\title{
151. Identification of Spectral Lines in the Violet Region of Three Carbon Stars HD 156074, HD 182040, and RU Cam
}

\author{
By Yoshio Fujita, M. J. A. \\ (Comm. Nov. 12, 1975)
}

It is very hard to get well exposed spectrograms of late-type carbon stars in the violet spectral region, because the intensity depletion is remarkable shortward beyond 24300 . In early-type carbon stars, however, we find the situation much promising. As we hardly find any identification table of spectral lines of carbon stars in this region, it seems to be worth-while to carry out such an identification program.

Fortunately during his stay at the Hale Observatories in 1972 as a guest investigator, the author could measure some coude spectrograms of three carbon stars HD 156074 (C1, 2), HD 182040 (C1, $2)$, and RU Cam $(\mathrm{C} 0,1)$ in the spectral region from $\lambda 3605$ to $\lambda 4350$. Although the wavelength region around $\mathrm{H}$ and $\mathrm{K}$ lines of $\mathrm{Ca}$ was too diffuse to carry on the accurate measurements, the other regions have been well exposed for measuring. The conversion of micrometer readings into wavelengths was carried out at the Computer Center, University of Tokyo. Identification was aided by Revised Multiplet Table (1945), Second Revision of Rowland Preliminary Table of Solar Spectrum Wavelength (1966), and Photometric Atlas of the Solar Spectrum (Utrecht, 1940). Table I gives the list of selected lines which are mostly free from blending. Identified elements in those stars are: Neutral; $\mathrm{Mg} \mathrm{Al} \mathrm{Si} \mathrm{Ca} \mathrm{Ti} \mathrm{V}$ Cr $\mathrm{Mn} \mathrm{Fe} \mathrm{Co} \mathrm{Ni.} \mathrm{Ionized;}$ Si Sc Ti V Cr Fe Sr Zr Ba La Nd Sm. CN $B^{2} \Sigma^{+}-X^{2} \Sigma^{+}(0,1)$ and $(1,2)$. $\mathrm{CH} A^{2} \Delta-X^{2} I I(0,0),(1,1)$, and $(2,2)$. In HD 156074 neutral lines of 4 th period elements $\mathrm{Ca}, \mathrm{Ti}, \mathrm{V}, \mathrm{Cr}, \mathrm{Mn}, \mathrm{Fe}$, and Co are enhanced. As for ionized elements, 4th period elements $\mathrm{Sc}, \mathrm{Ti}, \mathrm{V}, \mathrm{Cr}$, $\mathrm{Fe}$, and $\mathrm{Si}$ are weak in HD 156074 and rare-earth elements $\mathrm{Nd}$ and $\mathrm{Sm}$ are enhanced in HD 182040. Molecular band $\mathrm{CH}$ is enhanced in HD 156074 and $\mathrm{CN}$ is weak in RU Cam.

The author would like to thank the staff members of the Hale Observatories for their hospitality. His thanks are due to Prof. Y. Yamashita and Dr. T. Tsuji for their help to the computing program at the Computer Center, University of Tokyo and also to Miss K. Uzawa for preparing the Table. 
Table I

\begin{tabular}{|c|c|c|c|c|c|c|c|c|}
\hline $\begin{array}{c}\text { HD } 156074 \\
\lambda \\
\text { (measured) }\end{array}$ & Int. & $\begin{array}{c}\text { HD } 182040 \\
\lambda \\
\text { (measured) }\end{array}$ & Int. & $\begin{array}{c}\mathrm{RU} \text { Cam } \\
\lambda \\
\text { (measured) }\end{array}$ & Int. & Identification & \multicolumn{2}{|c|}{$\begin{array}{c}\text { Laboratory } \\
\lambda\end{array}$} \\
\hline & & . & & & & & & \\
\hline 3886.20 & 8 & 3885.75 & 7 & 3885.49 & 8 & $\mathrm{Fe} 4$ & 3886.28 & \\
\hline 86.95 & 8 & 86.55 & 7 & 86.43 & 8 & $\mathrm{Fe} 20$ & $\begin{array}{r}3000.20 \\
87.05\end{array}$ & \\
\hline 88.43 & 5 & 87.97 & 4 & 87.92 & $8(\mathrm{br})$ & $\mathrm{Fe} 45$ & 88.52 & \\
\hline 90.71 & 3 & 90.47 & 2 & 90.18 & 7 & $\mathrm{Fe} 280$ & 90.84 & \\
\hline 95.72 & 2 & 95.14 & 5 & 94.95 & 8 & Fe 4 & 95.66 & \\
\hline 97.24 & 4 & 96.93 & 2 & 96.78 & 8 & $\mathrm{Fe} 429$ & 97.45 & \\
\hline 3902.88 & 8 & 3902.52 & 6 & 3902.36 & 8 & $\mathrm{Fe} 45$ & 3902.95 & \\
\hline 04.66 & 2 & 04.41 & 1 & 04.08 & 7 & Ti 56 & 04.79 & \\
\hline 05.34 & 7 & 05.05 & 5 & 04.93 & 8 & $\mathrm{Si} 3$ & 05.53 & \\
\hline 06.36 & 8 & 05.95 & 5 & 05.70 & 8 & $\mathrm{Fe} 4$ & 06.48 & \\
\hline 09.70 & 4 & 09.35 & 3 & 09.24 & 8 & Fe 364 & 09.83 & \\
\hline 10.62 & 2 & 10.42 & 2 & 10.18 & $\begin{array}{l}0 \\
6\end{array}$ & Fe 284 & 10.85 & \\
\hline 13.40 & 5 & 12.90 & 5 & 12.87 & 7 & TiII 34 & 13.46 & \\
\hline 20.16 & 6 & 19.69 & 3 & 19.48 & 6 & $\mathrm{Fe} 4$ & 20.26 & \\
\hline 20.98 & 4 & 20.54 & 1 & 20.59 & 6 & Cr 23 & 21.02 & \\
\hline 22.79 & 8 & 22.34 & 3 & 22.21 & 6 & $\mathrm{Fe} 4$ & 22.91 & \\
\hline 47.49 & 3 & 47.01 & 2 & 46.95 & 5 & Fe 361,426 & 47.53 & \\
\hline 48.04 & 2 & 47.57 & 2 & 47.54 & 5 & $\mathrm{Fe} 562$ & 48.11 & \\
\hline 51.04 & 3 & 50.63 & 3 & 50.39 & 6 & Fe 661 Cr 136 & 51.16 & .10 \\
\hline 53.78 & 0 & 53.32 & 2 & 53.16 & 1 & $\mathrm{Fe} 362$ & 53.86 & .10 \\
\hline 55.14 & 2 & 54.74 & 3 & 54.69 & 7 & Fe 562 & 55.35 & \\
\hline 55.68 & 1 & 55.41 & 3 & 55.48 & 9 & $\mathrm{Fe} 488$ & 55.96 & \\
\hline 61.36 & 8 & 61.07 & 3 & 60.90 & 6 & AI I & 61.52 & \\
\hline 66.15 & 3 & 65.70 & 5 & 65.55 & 5 & $\mathrm{Fe} 45$ & 66.07 & \\
\hline 77.62 & 6 & 77.25 & 2 & 77.06 & 7 & Fe 72 & 77.74 & \\
\hline 78.42 & 5 & 78.02 & 2 & 77.90 & 7 & Fe 361 & 78.47 & \\
\hline 80.89 & 2 & 80.57 & 2 & 80.32 & 2 & $\mathrm{Fe} 122$ & 81.11 & \\
\hline 85.21 & 5 & 84.86 & 2 & 84.62 & 6 & Fe 661 & 85.39 & \\
\hline 86.04 & 5 & 85.69 & 2 & 85.48 & 6 & $\mathrm{Fe} 655$ & 86.18 & \\
\hline 88.39 & 4 & 87.99 & 4 & 87.85 & 6 & LaII 40 & 88.51 & \\
\hline 88.94 & 4 & 88.55 & 3 & 88.42 & 6 & ScII 8 & 89.06 & \\
\hline 90.99 & 2 & 90.67 & 3 & 90.52 & 4 & $\mathrm{Cr} 38 \mathrm{ZrII} 30$ & 91.12 & \\
\hline 95.95 & 2 & 95.37 & 2 & 95.30 & 7 & $\mathrm{Fe} 279$ & 96.00 & \\
\hline 98.52 & 7 & 98.12 & 5 & 98.10 & 8 & $\mathrm{Ti} 12$ & 98.64 & \\
\hline 98.98 & 3 & 98.60 & 6 & 98.46 & 8 & ZrII 16 & 98.98 & \\
\hline 4000.25 & 6 & 99.85 & 7 & 99.72 & 8 & Fe 426,556 & 4000.47 & .27 \\
\hline 01.48 & 4 & 4001.06 & 3 & 4000.95 & 4 & Fe 72 & 01.67 & \\
\hline 01.90 & 1 & 01.57 & 3 & 01.38 & 4 & FeII 29 & 02.07 & \\
\hline 02.87 & 3 & 02.42 & 5 & 02.23 & 6 & VII 9 & 02.94 & \\
\hline 05.55 & 1 & 05.15 & 7 & 04.97 & 9 & VII 32 & 05.71 & \\
\hline 07.13 & 3 & 06.85 & 4 & 06.73 & 5 & $\mathrm{Fe} 277$ & 07.28 & \\
\hline 11.26 & 2 & 11.02 & 2 & 10.77 & 2 & Fe 218 & 11.42 & \\
\hline 12.26 & 7 & 11.88 & 10 & 11.71 & 8 & TiII Il CeII 206 & 12.37 & .39 \\
\hline 14.44 & 4 & 14.00 & 6 & 13.85 & 8 & $\mathrm{Fe} 902 \operatorname{SCII} 8$ & 14.53 & .49 \\
\hline $\begin{array}{l}14.44 \\
16.26\end{array}$ & 3 & 16.00 & 0 & 15.83 & 1 & Fe 560 & 16.43 & \\
\hline 17.01 & 4 & 16.62 & 2 & 16.61 & 5 & Fe 279,527 & 17.10 & .16 \\
\hline 18.02 & 8 & 17.71 & 6 & 17.57 & 8 & Mn 5 & 18.10 & \\
\hline 20.79 & 6 & 20.41 & 2 & 20.20 & 3 & Co 16 & 20.90 & \\
\hline 23.20 & 1 & 22.98 & 1 & 22.72 & 6 & VII 32 & 23.39 & \\
\hline 25.02 & 4 & 24.66 & 7 & 24.53 & 7 & TiII 11 Ni 240 & 25.14 & .11 \\
\hline 28.27 & 3 & 27.94 & 7 & 27.64 & 7 & TiII 87 & 28.38 & .11 \\
\hline 30.55 & 8 & 30.23 & 8 & 30.11 & 9 & Mn 2 & 30.76 & \\
\hline 31.10 & 2 & 30.91 & 6 & 30.73 & 5 & $\mathrm{Fe} 486$ & 31.24 & \\
\hline 32.96 & 8 & 32.54 & 7 & 32.40 & 8 & Mn 2 & 33.07 & \\
\hline 34.36 & 8 & 34.00 & 7 & 33.81 & 8 & Mn $2 \mathrm{CH}(0,0) \mathrm{P} 17$ & 34.49 & .39 \\
\hline 36.59 & 1 & 36.18 & 2 & 36.02 & 1 & VII $9 \mathrm{CH}(1,1) \mathrm{RI}$ & 36.78 & .67 \\
\hline 40.53 & 7 & 40.19 & 7 & 40.08 & $\frac{1}{7}$ & $\mathrm{Fe} 655$ & 40.65 & \\
\hline 44.47 & 7 & 44.05 & 5 & 43.98 & 5 & $\mathrm{Fe} 359$ & 44.61 & \\
\hline 45.67 & 10 & 45.29 & 9 & 45.21 & 10 & $\mathrm{Fe} 43$ & 45.82 & \\
\hline 48.59 & 6 & 48.17 & 7 & 48.00 & 7 & Mn 5 ZrII 43 & 48.76 & .68 \\
\hline 49.20 & 4 & 48.92 & 3 & 48.84 & 4 & $\mathrm{Fe} 218$ & 49.34 & \\
\hline 50.19 & 3 & 49.76 & 2 & 49.82 & 4 & $\operatorname{ZrII} 43$ & 50.32 & \\
\hline 51.00 & 4 & 50.61 & 7 & 50.41 & 5 & VII 32 & 51.05 & \\
\hline 51.76 & 4 & 51.48 & 6 & 51.33 & 5 & CrII $19 \mathrm{Fe} 700$ & 51.92 & \\
\hline 54.76 & 6 & 54.39 & 5 & 54.24 & 7 & $\mathrm{Fe} 698$ & 54.83 & .88 \\
\hline 55.43 & 6 & 55.01 & 5 & 54.88 & 6 & Mn 5 & 55.54 & \\
\hline 57.32 & 6 & 56.97 & 6 & 56.84 & 7 & $\mathrm{Mg} 16 \mathrm{Fe} 277$ & 57.51 & .36 \\
\hline 60.97 & 2 & 60.63 & 5 & 60.45 & 4 & NaII 10 & 61.09 & \\
\hline 61.71 & 2 & 61.37 & 3 & 61.19 & 0 & Mn 29 & 61.74 & \\
\hline 62.41 & 7 & 62.05 & 6 & 61.83 & 4 & Fe 359 & 62.45 & \\
\hline 63.48 & 10 & 63.07 & 9 & 62.87 & 9 & $\mathrm{Fe} 43$ & 63.60 & \\
\hline 65.17 & 5 & 64.85 & 4 & 64.60 & 4 & Fe 698 & 65.40 & \\
\hline 66.88 & 5 & 66.53 & 3 & 66.52 & 6 & Fe 358 & 66.98 & \\
\hline 67.87 & 6 & 67.59 & 4 & 67.33 & 3 & Fe 559 & 67.98 & \\
\hline 68.97 & 5 & 68.63 & $\begin{array}{l}4 \\
4\end{array}$ & 68.37 & 2 & Fe 557 & 69.08 & \\
\hline 70.12 & 5 & 69.78 & $i$ & 69.57 & 1 & Mn 5 & 70.28 & \\
\hline 70.68 & 6 & 70.44 & 7 & 70.22 & 5 & $\mathrm{Fe} 558$ & 70.77 & \\
\hline 71.57 & 10 & 71.14 & 9 & 71.06 & $\begin{array}{l}3 \\
8\end{array}$ & Fe 43 & 71.74 & \\
\hline 74.63 & 7 & 74.20 & 5 & 74.09 & 5 & Fe 524 & 74.79 & \\
\hline 77.65 & 8 & 77.23 & 9 & 77.11 & 8 & SrII I & 77.71 & \\
\hline
\end{tabular}


Table I (Continued)

\begin{tabular}{|c|c|c|c|c|c|c|c|c|}
\hline $\begin{array}{c}\text { HD } 156074 \\
\lambda \\
\text { (measured) }\end{array}$ & Int. & $\begin{array}{c}\text { HD } 182040 \\
\lambda \\
\text { (measured) }\end{array}$ & Int. & $\begin{array}{c}\text { RU Cam } \\
\lambda \\
\text { (measured) }\end{array}$ & Int. & Identification & \multicolumn{2}{|c|}{$\begin{array}{c}\text { Laboratory } \\
\lambda\end{array}$} \\
\hline 4079.20 & 8 & 4078.77 & 8 & 4078.64 & 5 & Mn 5 & 4079.24 & .42 \\
\hline 79.72 & 6 & 79.30 & 5 & 79.14 & 3 & Fe 359 & 79.85 & \\
\hline 80.07 & 6 & 79.79 & 4 & 79.57 & 4 & Fe 558 & 80.23 & \\
\hline 82.14 & 5 & 81.80 & 7 & 81.59 & $4(\mathrm{br})$ & $\mathrm{Fe} 698$ & 82.13 & \\
\hline 82.91 & 6 & 82.54 & 7 & 82.41 & 5 & Mn 5 & 82.94 & \\
\hline 84.35 & 5 & 83.97 & 6 & 83.89 & 3 & $\mathrm{Fe} 698$ & 84.50 & \\
\hline 85.23 & 5 & 84.78 & 5 & 84.58 & 5 & Fe 559 CeII 172 & 85.31 & .23 \\
\hline 86.54 & 2 & 86.14 & 5 & 86.00 & 3 & LaII 10 & 86.72 & 0 \\
\hline 86.97 & 1 & 86.64 & 5 & 86.45 & 3 & $\mathrm{Fe} 694$ & 87.10 & \\
\hline 89.97 & 6 & 89.69 & 4 & 89.42 & 2 & $\mathrm{Fe} 700$ & 90.09 & \\
\hline 90.68 & 4 & 90.34 & 4 & 90.27 & 2 & $\mathrm{Fe} 695$ & 90.98 & \\
\hline 91.41 & 3 & 91.03 & 4 & 90.89 & 0 & $\mathrm{Fe} 357$ & 91.56 & \\
\hline 92.30 & 8 & 91.88 & 7 & 91.90 & $6(b r)$ & Co 29 & 92.39 & \\
\hline 95.89 & 7 & 95.49 & 7 & 95.30 & 5 & Fe 217 & 95.98 & \\
\hline 98.04 & 4 & 97.64 & 5 & 97.68 & 3 & $\mathrm{Fe} 558$ & 98.18 & \\
\hline 98.94 & 7 & 98.47 & 7 & 98.36 & 3 & $\mathrm{Fe} 600,651$ & 99.08 & \\
\hline 99.74 & 5 & 99.37 & 6 & 99.33 & 2 & V 27 & 99.80 & \\
\hline 4100.56 & 7 & 4100.19 & 7 & 99.96 & 3 & $\mathrm{Fe} 18$ & 4100.75 & \\
\hline 01.24 & 5 & 00.98 & 7 & 4100.73 & 3 & $\mathrm{Fe} 698$ & 01.27 & \\
\hline 01.96 & 0 & 01.57 & 6 & 01.53 & 2 & V 41 & 02.16 & \\
\hline 02.62 & 5 & 02.23 & 7 & 02.12 & 2 & $\mathrm{Si} 2 \mathrm{Ni} 255 \mathrm{P}$ & 02.93 & .74 \\
\hline 03.38 & 5 & 02.94 & 7 & 02.82 & 1 & $\mathrm{CN}(1,2)$ R58 & 03.46 & \\
\hline 04.77 & $7(b r)$ & 04.48 & 7 & 04.24 & 5 & Fe 694 & 04.97 & \\
\hline 06.31 & 7 & 06.00 & 8 & 05.78 & 5 & $\mathrm{Fe} 697$ & 06.44 & \\
\hline 07.42 & 6 & 06.88 & 8 & 06.83 & 5 & $\mathrm{Fe} 354$ & 07.49 & \\
\hline 07.99 & 5 & 07.65 & 7 & 07.61 & 4 & Fe 559 & 08.13 & \\
\hline 08.94 & 4 & 08.65 & 7 & 08.47 & 4 & $\mathrm{Fe} 558$ & 09.07 & \\
\hline 10.43 & 8 & 10.06 & $7(b r)$ & 09.95 & 3 & Co 29 & 10.53 & \\
\hline 11.39 & 7 & 10.93 & 7 & 10.75 & 1 & Cr 97 & 11.36 & \\
\hline 11.71 & 7 & 11.23 & 7 & 11.11 & 2 & V 27 & 11.79 & \\
\hline 12.95 & 7 & 12.56 & 7 & 12.38 & 3 & $\mathrm{Fe} 1103$ & 12.97 & \\
\hline 14.49 & 7 & 14.03 & 6 & 13.82 & 3 & $\mathrm{Fe} 357$ & 14.45 & \\
\hline 15.06 & 6 & 14.74 & 5 & 14.51 & 2 & V 27 & 15.19 & \\
\hline 16.36 & 6 & 16.05 & 5 & 15.80 & 3 & V 27 & 16.47 & \\
\hline 17.61 & 7 & 17.23 & 3 & 17.14 & 3 & $\mathrm{Fe} 700,1103$ & 17.87 & \\
\hline 21.04 & 4 & 20.76 & 2 & 20.61 & 5 & Co 28 & 21.32 & \\
\hline 21.66 & 2 & 21.25 & $i$ & 21.13 & 1 & Fe 356 & 21.81 & \\
\hline 23.55 & 4 & 23.22 & 1 & 23.12 & 6 & Fe 217,422 & 23.75 & \\
\hline 25.82 & 7 & 25.50 & 6 & 25.35 & $6(\mathrm{br})$ & $\mathrm{Fe} 354$ & 25.88 & \\
\hline 28.59 & 1 & 28.17 & 1 & 28.08 & 4 & FeII 27 & 28.74 & \\
\hline 30.05 & 7 & 29.64 & 6 & 29.51 & 5 & $\mathrm{Fe} 44,486$ & 30.04 & \\
\hline 30.58 & 6 & 30.13 & 4 & 30.13 & $7(b r)$ & BaII 4 & 30.65 & \\
\hline 31.86 & 9 & 31.41 & 7 & 31.35 & $8(\mathrm{br})$ & $\mathrm{Fe} 43$ & 32.06 & \\
\hline 36.41 & 6 & 35.99 & 2 & 35.85 & 2 & $\mathrm{Fe} 694$ & 36.51 & \\
\hline 37.07 & 7 & 36.65 & 3 & 36.60 & 3 & $\mathrm{Fe} 726$ & 37.00 & \\
\hline 43.41 & 4 & 42.90 & 2 & 42.76 & 4 & $\mathrm{Fe} 523$ & 43.42 & \\
\hline 43.78 & 7 & 43.37 & 3 & 43.23 & $5(d)$ & $\mathrm{Fe} 43$ & 43.87 & \\
\hline 45.87 & 4 & 45.43 & 3 & 45.24 & 5 & $\mathrm{Fe} 422$ & 46.07 & \\
\hline 47.57 & 5 & 47.12 & 1 & 47.02 & 5 & $\mathrm{Fe} 42$ & 47.67 & \\
\hline 48.52 & 3 & 48.08 & 2 & 47.91 & 1 & Cr 241 & 48.52 & \\
\hline 49.09 & 2 & 48.64 & 1 & 48.57 & 2 & $\operatorname{ZrII} 41$ & 49.22 & \\
\hline 52.19 & 5 & 51.64 & 2 & 51.49 & 5 & $\mathrm{Fe} 18$ & 52.17 & \\
\hline 53.78 & 3 & 53.33 & 3 & 53.24 & 3 & Fe 695 & 53.91 & \\
\hline 54.53 & 6 & 54.11 & 3 & 53.96 & 5 & $\mathrm{Fe} 355$ & 54.50 & \\
\hline 55.22 & 2 & 54.77 & 2 & 54.67 & 1 & SmII 8, 50 & 55.22 & \\
\hline 56.70 & 2 & 56.26 & 4 & 56.16 & 5 & $\mathrm{Fe} 354$ & 56.80 & \\
\hline 58.62 & 2 & 58.23 & 4 & 58.28 & 3 & $\mathrm{Fe} 695$ & 58.80 & \\
\hline 62.43 & 5 & 62.02 & $7(\mathrm{br})$ & 61.90 & 3 & $\mathrm{CH}(0,0) \mathrm{R} 25$ & 62.46 & .66 \\
\hline 63.34 & 6 & 62.95 & 7 & 62.88 & 7 & TiII 105 & 63.64 & 80 \\
\hline 63.93 & 5 & 63.61 & 6 & 63.39 & 7 & VII 37 & 64.02 & \\
\hline 65.99 & 5 & 65.62 & 5 & 65.45 & 4 & BaII 4 & 66.00 & \\
\hline 67.18 & 6 & 66.71 & 7 & 66.70 & 6 & $\operatorname{Mg~} 15$ & 67.26 & \\
\hline 68.48 & 6 & 68.05 & 8 & 67.93 & 2 & $\mathrm{Fe} 689$ & 68.63 & \\
\hline 73.30 & 7 & 72.96 & 5 & 72.86 & 5 & FeII 27 & 73.45 & \\
\hline 74.80 & 8 & 74.35 & 3 & 74.33 & 3 & $\mathrm{Fe} 19$ & 74.92 & \\
\hline 80.69 & 7 & 80.30 & 7 & 80.19 & 2 & $\mathrm{CH}(0,0) \mathrm{R} 21$ & 80.81 & \\
\hline 83.26 & 6 & 82.97 & 6 & 82.92 & 3 & VII 87 & 83.44 & \\
\hline 84.10 & 6 & 83.64 & 8 & 83.56 & 3 & TiII 21 & 84.33 & \\
\hline 84.80 & 7 & 84.40 & 7 & 84.24 & 3 & Fe 355 & 84.90 & \\
\hline 86.94 & 7 & 86.58 & 6 & 86.42 & 2 & $\mathrm{Fe} 152$ & 87.04 & \\
\hline 87.64 & 8 & 87.24 & 8 & 87.15 & 2 & Fe 152 & 87.80 & \\
\hline 89.30 & 7 & 88.96 & 7 & 88.85 & $\overline{1}$ & $\mathrm{Fe} 940$ & 89.56 & \\
\hline 89.99 & 8 & 89.59 & 7 & 89.40 & 2 & Cr 84 & 90.16 & \\
\hline 90.66 & 6 & 90.28 & 5 & 90.07 & 1 & Co 1 & 90.71 & \\
\hline 92.32 & 7 & 91.90 & 6 & 91.78 & $\overline{1}$ & $\mathrm{CH}(0,0) \mathrm{R} 19$ & 92.57 & \\
\hline 94.14 & 2 & 93.77 & 4 & 93.61 & $\mathrm{I}$ & 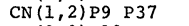 & 94.24 & \\
\hline 94.64 & 7 & 94.31 & 4 & 94.12 & 3 & $\mathrm{CH}(0,0) \mathrm{Rl9}$ & 94.85 & \\
\hline 96.34 & 7 & 96.00 & 6 & 95.96 & 6 & $\mathrm{Fe} 418$ & 96.53 & \\
\hline 98.05 & 8 & 97.59 & 7 & 97.63 & 6 & $\mathrm{Fe} 152,693$ & 98.31 & .27 \\
\hline 99.00 & 6 & 98.61 & 7 & 98.46 & 5 & Fe 522 & 99.10 & \\
\hline
\end{tabular}


Table I (Continued)

\begin{tabular}{|c|c|c|c|c|c|c|c|c|}
\hline $\begin{array}{c}\text { HD } 156074 \\
\lambda \\
\text { (measured) }\end{array}$ & Int. & $\begin{array}{c}\text { HD } 182040 \\
\lambda \\
\text { (measured) }\end{array}$ & Int. & $\begin{array}{c}\text { RU Cam } \\
\lambda \\
\text { (measured) }\end{array}$ & Int. & Identification & \multicolumn{2}{|c|}{$\underset{\lambda}{\stackrel{\text { Laboratory }}{ }}$} \\
\hline 4199.86 & 7 & 4199.37 & 6 & 4199.32 & 4 & $\mathrm{Fe} 3$ & 4199.97 & \\
\hline 4201.94 & 9 & 4201.44 & 6 & 4201.34 & 7 & $\mathrm{Fe} 42$ & 4202.03 & \\
\hline 03.76 & 6 & 03.56 & 6 & 03.30 & 3 & $\mathrm{Fe} 355$ & 03.99 & \\
\hline 05.91 & 5 & 05.51 & 4 & 05.25 & 0 & $\mathrm{CN}(0,1) \mathrm{P} 48$ & 05.89 & .96 \\
\hline 06.46 & 7 & 06.18 & 5 & 05.97 & 2 & $\mathrm{Fe} 3 \mathrm{CH}(0,0) \mathrm{RI} 7$ & 06.70 & .58 \\
\hline 08.09 & 6 & 07.57 & 4 & 07.49 & 1 & $\mathrm{CH}(0,1)$ P 45 & 08.10 & .17 \\
\hline 09.43 & 6 & 08.98 & 4 & 08.87 & 1 & $\mathrm{CH}(1,1) \mathrm{Rl9}$ & 09.50 & .60 \\
\hline 11.12 & 7 & 10.64 & 4 & 10.59 & 1 & $\mathrm{Cr} 133$ & 11.35 & \\
\hline 11.66 & 7 & 11.22 & 5 & 11.15 & 1 & $\mathrm{Ti} 279$ & 11.73 & \\
\hline 12.19 & 6 & 11.68 & 4 & 11.68 & 0 & $\mathrm{CN}(0,1)$ P 38 & 12.23 & \\
\hline 13.46 & 5 & 13.01 & 2 & 13.04 & 2 & $\mathrm{Fe} 355$ & 13.65 & \\
\hline 14.95 & 6 & .14 .70 & 10 & 14.50 & 8 & $\mathrm{CN}(0,1)$ P $15 \quad \mathrm{P} 30$ & 15.17 & \\
\hline 22.10 & 4 & 21.78 & 6 & 21.62 & 3 & $\mathrm{Fe} 152$ & 22.22 & \\
\hline 24.04 & 3 & 23.67 & 5 & 23.57 & 3 & Fe 689 & 24.18 & \\
\hline 25.22 & 6 & 24.78 & 7 & 24.62 & 4 & VII 37 & 25.23 & \\
\hline 26.55 & 10 & 26.11 & 10 & 26.03 & 8 & $\mathrm{Ca} 2$ & 26.73 & \\
\hline 33.89 & 2 & 33.57 & 1 & 33.51 & 2 & $\mathrm{~V} 6,111$ & 34.00 & \\
\hline 35.05 & 6 & 34.65 & 7 & 34.55 & 3 & Mn 23 & 35.14 & \\
\hline 35.75 & 9 & 35.31 & 9 & 35.31 & 9 & $\mathrm{Fe} 152$ & 35.94 & \\
\hline 38.71 & 5 & 38.29 & 4 & 38.22 & 3 & Fe 693 & 38.82 & \\
\hline 40.24 & 2 & 39.89 & 2 & 39.84 & 1 & $\mathrm{Fe} 764$ & 40.37 & \\
\hline 43.33 & 6 & 42.84 & 3 & 42.70 & 4 & $\mathrm{CH}(0,0) \mathrm{R} 11$ & 43.37 & \\
\hline 45.16 & 7 & 44.72 & 5 & 44.60 & 3 & $\mathrm{Fe} 352$ & 45.26 & \\
\hline 45.89 & 6 & 45.43 & 5 & 45.38 & 3 & Fe 906 & 46.09 & \\
\hline 46.72 & 6 & 46.28 & 9 & 46.19 & 5 & ScII 7 & 46.83 & \\
\hline 47.30 & 6 & 46.82 & 5 & 46.74 & 7 & Fe 693 & 47.43 & \\
\hline 48.18 & 5 & 47.71 & 5 & 47.67 & 4 & Fe 482 & 48.23 & \\
\hline 48.73 & 3 & 48.18 & 1 & 48.06 & 4 & $\mathrm{CH}(0,0) \mathrm{R} 10$ & 48.73 & .94 \\
\hline 49.40 & 5 & 48.88 & 1 & 48.88 & 2 & $\mathrm{CH}(0,0) \mathrm{R} 10$ & 49.49 & .64 \\
\hline 50.00 & 7 & 49.58 & 7 & 49.49 & 6 & $\mathrm{Fe} 152$ & 50.13 & \\
\hline 50.67 & 8 & 50.22 & 9 & 50.16 & 6 & Fe 42 & 50.79 & \\
\hline 54.20 & 6 & 53.84 & 6 & 53.71 & 4 & $\mathrm{Cr} 1$ & 54.35 & \\
\hline 58.17 & 8 & 57.69 & 7 & 57.63 & 7 & $\mathrm{Fe} 3$ & 58.32 & \\
\hline 62.54 & 1 & 62.08 & 1 & 61.95 & 0 & $\mathrm{CH}(2,2) \mathrm{R} 12$ & 62.59 & .71 \\
\hline 64.10 & 1 & 63.73 & 1 & 63.57 & 1 & $\mathrm{Fe} 692$ & 64.21 & \\
\hline 67.76 & 4 & 67.30 & 5 & 67.30 & 5 & $\mathrm{Fe} 482 \mathrm{CH}(0,0) \mathrm{R} 7$ & 67.83 & .75 \\
\hline 68.62 & 2 & 68.24 & 6 & 68.24 & 3 & Fe 649 & 68.74 & \\
\hline 70.08 & 1 & 69.65 & 1 & 69.46 & 2 & Ti 251 CH $(1,1) R 7$ & 70.17 & \\
\hline 71.10 & 9 & 70.58 & 6 & 70.60 & 7 & Fe 152 & 71.16 & \\
\hline 71.65 & 10 & 71.22 & 9 & 71.16 & 7 & Fe 42 & 71.76 & \\
\hline 74.69 & 7 & 74.25 & 6 & 74.20 & 6 & $\mathrm{Cr} 1$ & 74.80 & \\
\hline 78.76 & $i$ & 78.27 & 0 & 78.29 & 1 & $\mathrm{CH}(0,0) Q 25$ & 78.85 & \\
\hline 79.68 & $5(b r)$ & 79.29 & 2 & 79.17 & 6 & $\mathrm{CH}(0,0)$ R5 Q23 & 79.72 & \\
\hline 80.86 & 5 & 80.41 & 1 & 80.39 & 6 & $\mathrm{CH}(0,0) Q 24 \quad(1,1) \mathrm{R} 5$ & 80.96 & \\
\hline 82.33 & 6 & 81.82 & 6 & 81.85 & 7 & $\mathrm{Fe} 71$ & 82.41 & \\
\hline 82.83 & 5 & 82.49 & 4 & 82.43 & 6 & $\mathrm{Ca} 5$ & 83.01 & \\
\hline 84.11 & 4 & 83.59 & 2 & 83.73 & 3 & $\mathrm{CH}(0,0) Q 21$ & 84.23 & \\
\hline 87.74 & 7 & 87.31 & 8 & 87.33 & 5 & TiII 20 & 87.89 & \\
\hline 89.18 & 3 & 88.78 & 2 & 88.75 & 3 & $\mathrm{Ca} 5$ & 89.36 & \\
\hline 89.62 & 7 & 89.25 & 5 & 89.00 & 4 & $\mathrm{Cr} 1$ & 89.72 & \\
\hline 90.10 & 6 & 89.70 & 7 & 89.59 & 5 & TiII 41 & 90.22 & \\
\hline 90.79 & 6 & 90.38 & 4 & 90.34 & 3 & $\mathrm{Ti} 44 \mathrm{Fe} 351$ & 90.93 & .87 \\
\hline 91.37 & 6 & 90.89 & 2 & 90.74 & 3 & $\mathrm{Fe} 3,41$ & 91.47 & 8 \\
\hline 92.95 & 4 & 92.57 & 2 & 92.59 & 3 & $\mathrm{CH}(0,0)$ Q18 Q17 & 93.04 & .11 \\
\hline 94.00 & 9 & 93.51 & 9 & 93.35 & 8 & $\mathrm{Fe} 41$ & 94.13 & \\
\hline 94.78 & 3 & 94.30 & 7 & 94.09 & 8 & SiII 15 & 94.77 & \\
\hline 99.90 & 6 & 99.49 & 6 & 99.28 & 5 & TiII 41 & 4300.05 & \\
\hline 4300.41 & 5 & 4300.01 & 1 & 99.87 & 8 & $\mathrm{Ti} 44$ & 00.57 & \\
\hline 02.50 & 5 & 01.91 & 5 & 4301.87 & 5 & Ca 5 & 02.53 & \\
\hline 03.03 & 4 & 02.65 & 7 & 02.43 & 5 & FeII 27 & 03.17 & \\
\hline 07.77 & 10 & 07.32 & 9 & 07.50 & 8 & $\mathrm{Fe} 42$ & 07.91 & \\
\hline 09.39 & 3 & 08.94 & 8 & 08.82 & 3 & $\mathrm{Fe} 4,14$ & 09.38 & \\
\hline 12.68 & 5 & 12.30 & 8 & 12.08 & 5 & TIII $41 \mathrm{CH}(0,0) Q 4$ & 12.86 & \\
\hline 14.01 & 8 & 13.59 & 9 & 13.40 & 6 & ScII 15 & 14.08 & \\
\hline 16.78 & 1 & 16.25 & 2 & 16.21 & 2 & TiII 94 & 16.81 & \\
\hline 17.32 & 0 & 16.75 & 2 & 16.59 & 2 & $\operatorname{ZrII} 40$ & 17.32 & \\
\hline 18.57 & 5 & 18.14 & 6 & 18.03 & 4 & $\mathrm{Ca} 5$ & 18.65 & \\
\hline 20.68 & 6 & 20.25 & 8 & 20.10 & $7(\mathrm{br})$ & ScII 15 & 20.75 & \\
\hline 24.87 & 4 & 24.40 & 8 & 24.20 & 7 & ScII 15 & 25.01 & \\
\hline 25.66 & 9 & 25.19 & 8 & 24.91 & 9 & $\mathrm{Fe} 42$ & 25.77 & \\
\hline 30.21 & 1 & 29.69 & 6 & 29.71 & $4(\mathrm{br})$ & TiII 94 & 30.26 & \\
\hline 31.53 & 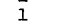 & 31.25 & 5 & 30.93 & 2 & $\mathrm{Ni} 52$ & 31.65 & \\
\hline 33.69 & 1 & 33.19 & 2 & 33.17 & 2 & LaII 24 & 33.76 & \\
\hline 36.97 & 6 & 36.47 & 5 & 36.42 & 6 & $\mathrm{Fe} 41$ & 37.05 & \\
\hline 39.36 & 5 & 38.98 & 5 & 38.74 & 5 & Cr 22 & 39.45 & \\
\hline 44.25 & 3 & 43.81 & 8 & 43.63 & 5 & TiII 20 & 44.29 & \\
\hline 46.44 & 2 & 46.01 & 3 & 45.81 & 1 & $\mathrm{Fe} 598$ & 46.56 & \\
\hline 47.75 & 2 & 47.23 & 3 & 47.15 & I & $\mathrm{Fe} 828$ & 47.85 & \\
\hline
\end{tabular}

In case of two contributors, the preceding element is the dominant one. HD 156074: Ce 12737, Ce 8062

HD 182040: Ce 3103

RU Cam: Pc 2919 